\title{
Gene therapy by allele selection in a mouse model of beta-thalassemia
}

\author{
Sigrid Eckardt, ${ }^{1}$ N. Adrian Leu, ${ }^{2}$ Ashley Yanchik, ${ }^{2}$ Seigo Hatada, ${ }^{3}$ \\ Michael Kyba, ${ }^{4}$ and K. John McLaughlin ${ }^{1,5}$
}

\begin{abstract}
${ }^{1}$ Center for Molecular and Human Genetics, The Research Institute at Nationwide Children's Hospital, Columbus, Ohio, USA. 2University of Pennsylvania School of Veterinary Medicine, Philadelphia, Pennsylvania, USA. ${ }^{3}$ Department of Pathology and Laboratory Medicine, University of North Carolina, Chapel Hill, North Carolina, USA. ${ }^{4}$ Lillehei Heart Institute and Department of Pediatrics, University of Minnesota, Minneapolis, Minnesota, USA. 5Department of Pediatrics, College of Medicine, The Ohio State University, Columbus, Ohio, USA.
\end{abstract}

\begin{abstract}
To be of therapeutic use, autologous stem cells derived from patients with inherited genetic disorders require genetic modification via gene repair or insertion. Here, we present proof of principle that, for diseases associated with dominant alleles (gain-of-function or haploinsufficient loss-of-function), disease allele-free ES cells can be derived from afflicted individuals without genome manipulation. This approach capitalizes on the derivation of uniparental cells, such as parthenogenetic (PG) ES cell lines from disease allele-free gametes. Diploid mammalian uniparental embryos with only maternally (oocyte-) or paternally (sperm-)derived genomes fail early in development due to the nonequivalence of parental genomes caused by genomic imprinting. However, these uniparental embryos develop to the blastocyst stage, allowing the derivation of ES cell lines. Using a mouse model for dominant beta-thalassemia, we developed disease allele-free PG ES cell lines from the oocytes of affected animals. Phenotype correction was obtained in donor-genotype recipients after transplantation of in vitro hematopoietic ES cell derivatives. This genetic correction strategy without gene targeting is potentially applicable to any dominant disease. It could also be the sole approach for larger or more complex mutations that cannot be corrected by homologous recombination.
\end{abstract}

\section{Introduction}

Potential therapeutic strategies for the treatment of inherited genetic disorders include gene therapy, stem cell-based transplantation approaches, and a combination thereof. Gene therapy via the introduction of a therapeutic gene into target tissues, typically by virus-mediated transfer, has, despite its simple concept, proven difficult with respect to obtaining or maintaining curative gene expression levels, ensuring tissue specificity, and avoiding side effects such as genotoxicity and insertional activation $(1,2)$. Genetic repair by homologous recombination in stem cells, particularly ES cells and induced pluripotent stem (iPS) cells derived from somatic cells, which are then utilized for tissue repair, provides another perspective for disease treatment. Proof of principle for this concept has been demonstrated in the mouse to repair the immune deficiency-causing Rag2 mutation in ES cells derived by somatic cell nuclear transfer (3) and treat sickle cell anemia via derivation and genetic repair of iPS cells (4). However, gene repair or transgene insertion by homologous recombination, which is easily achieved in mouse, has proven considerably more challenging in human pluripotent cells (5). Here, we describe an approach to gene repair that capitalizes on the derivation of uniparental, such as parthenogenetic (PG), ES cell lines from the gametes of an afflicted individual. Mammalian uniparental embryos with only maternally (oocyte-) or paternally (sperm-)derived genomes fail early in development $(6,7)$, due to the nonequivalence of parental genomes caused by genomic imprinting (8). However, diploid uniparental embryos frequently develop to the blastocyst stage and can give rise to ES cell lines (9, 10). As half of the gametes from patients with genetically dominant diseases will not contain the mutant allele, the derivation of PG

Conflict of interest: The authors have declared that no conflict of interest exists. Citation for this article: J Clin Invest. 2011;121(2):623-627. doi:10.1172/JCI45377.
ES cell lines from patient gametes could provide a source of disorder-free, autologous cells for tissue repair; for example, in patients with blood disorders resulting from autosomal dominance or haploinsufficiency (11-16) or with autosomal dominant cancer predisposition genes (17). PG ES derivation in the mouse can be very efficient (18); and initial protocols from several laboratories have established the practicality of deriving human PG ES cell lines (19, 20). PG ES cells can be MHC homozygous or can contain the full MHC complement of a heterozygous oocyte donor $(18,19,21)$.

To demonstrate the feasibility of producing and using uniparental ES cell lines according to this concept, we pursued the derivation and therapeutic utilization of PG ES cell lines in a mouse model for a dominant thalassemia. Mice heterozygous for a deletion of both adult globin genes (Hbb-b1 and $H b b-b 2$ genes; mutant allele termed $H b b^{\text {th- }}$ ) are phenotypically anemic, with low hematocrit and red blood cell count, prominent anisocytosis and poikilocytosis in blood smear preparations, high white cell count, splenomegaly, and iron deposits in several organs and have been a model for the more severe forms of human thalassemia intermedia (22). Our previous data had demonstrated successful tissue repair by uniparental, including PG, cells derived from WT donors in the hematopoietic system (23). Here, we provide proof of principle for gene repair without genome modification by the derivation of PG ES cells from a thalassemic ( $H b b^{\text {th. }}$ heterozygous) mouse, identification of PG ES cell lines without a mutation-bearing allele, and correction of the thalassemic phenotype in oocyte donor-genotype mice after transplantation of in vitro hematopoietic derivatives.

\section{Results and Discussion}

We derived PG ES cell lines for therapeutic utilization in a mouse model for a dominant thalassemia as outlined in Figure 1. Unfertilized metaphase II (MII) oocytes from $H b b^{t b} \cdot 4$ heterozygous (C57BL/6 


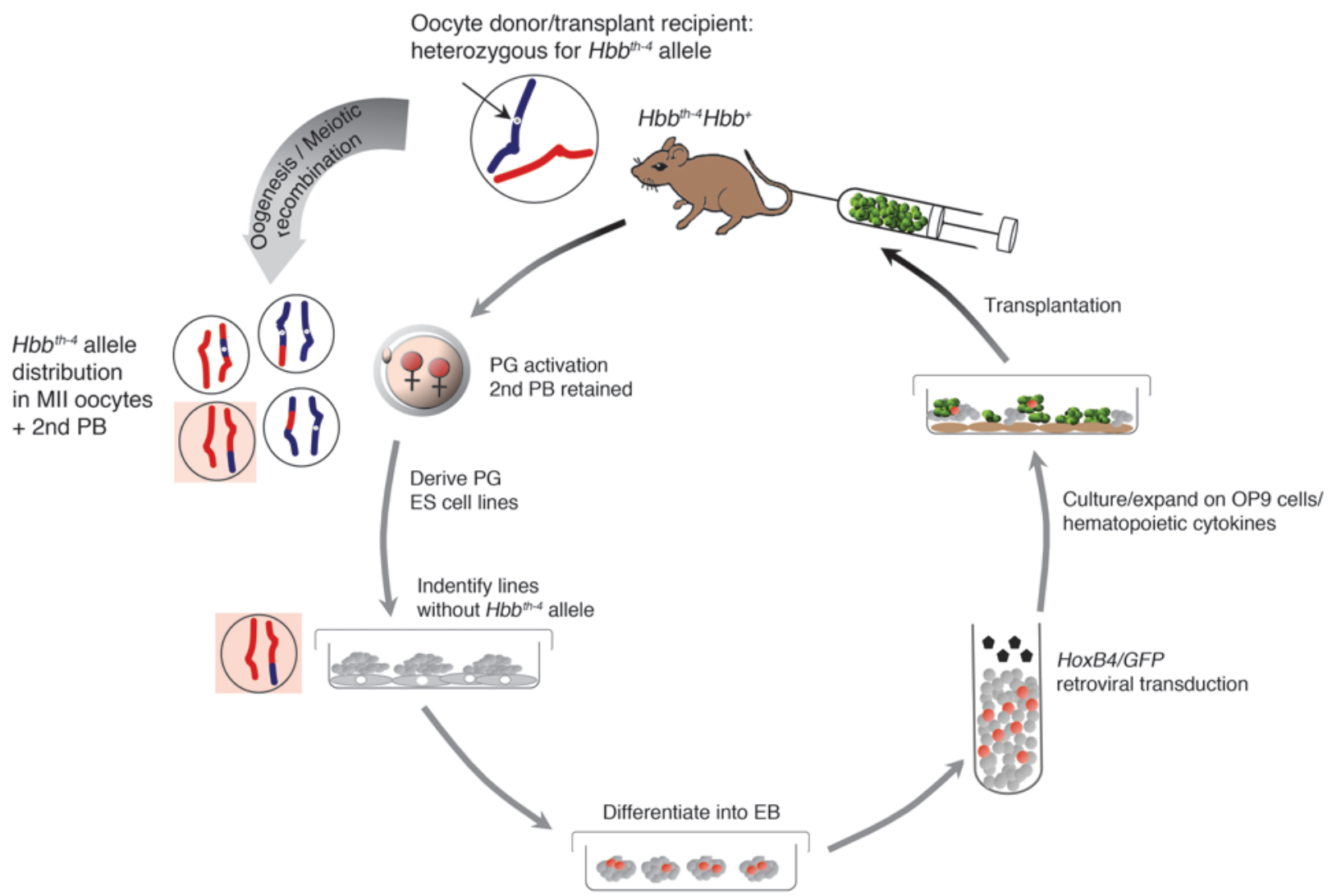

Figure 1

Derivation and therapeutic utilization of PG ES cells. Experimental design. ES cell lines derived from $H b b^{\text {th- } 4}$ allele-free PG zygotes generated from oocytes of $\mathrm{Hbb}^{\text {th-4 }}$ heterozygous mice are differentiated into hematopoietic progenitor/stem cells in vitro and transplanted into donor-genotype recipients. Simplified representation of chromosomes carrying normal (red) and $\mathrm{Hbb}^{\text {th-4 }}$ (blue, thin arrow) alleles; potential recombination illustrated by color. PB, polar body, EB, embryoid body.

$\times$ 129S1) $\mathrm{F}_{1}$ (designated B6129S1F1) female mice were chemically activated in the presence of cytochalasin $\mathrm{B}$, a drug that prevents second polar body extrusion. The resulting parthenogenetic zygotes were diploid, with 2 copies of the adult hemoglobin locus, i.e., either homozygous for the normal $H b b$ allele ( $H b b-b 1$ and $H b b-b 2$ genes; termed $\left.\mathrm{Hbb}^{+}\right), \mathrm{Hbb}^{t h-4}$ homozygous, or $\mathrm{Hbb}^{+}$and $\mathrm{Hbb}^{t b-4}$ heterozygous (Figure 1 and Supplemental Figure 1; supplemental material available online with this article; doi:10.1172/JCI45377DS1). ES cells derived from PG zygotes not retaining the $\mathrm{Hbb}^{\text {th-4 }}$ allele were then differentiated into hematopoietic progenitor/stem cells in vitro and transplanted into donor-genotype recipients.

In PG ES cells rendered diploid via second polar body retention, the frequency of obtaining mutant allele-free uniparental embryos from heterozygous donors depends on the probability of meiotic recombination at the desired locus, which is associated with the distance from the centromere $(18,24)$. For centromerically proximal genes, the frequency is predicted to be $50 \%$, while for distal genes, the frequency approaches $25 \%$ (with $50 \%$ heterozygous and $25 \%$ homozygous mutant). Of 13 PG ES cell lines derived from $\mathrm{Hbb}^{t h-4}$ heterozygous females, 3 were $H b b^{t h-4}$ allele-free, none homozygous, and 10 heterozygous for the allele (Table 1 and Supplemental Figure $2 \mathrm{~A}$ ), consistent with recombination expected at the murine $\mathrm{Hbb}$ locus located relatively centrally on chromosome 7, $50 \mathrm{cM}$ from the centromere. Two Hbbth-4 allele-free PG ES cell lines with normal chromosome counts (Supplemental Figure 2B) were additionally genotyped for MHC homo- or heterozygosity utilizing SNPs specific

Table 1

Derivation of $H b b^{\text {th-4 }}$ allele-free PG ES cell lines from $H b b^{\text {th-4 }} / H b b^{+}$heterozygous females

\section{Oocyte genotype \\ $\mathrm{B} 6129 \mathrm{~S} 1 \mathrm{~F} 1 \mathrm{Hbb^{+ } / H b b ^ { + }}$ (WT) B6129S1F1 $H b b^{\text {th- }-4 / H b b^{+}}$ (heterozygous) $)^{\mathrm{A}}$}

$\begin{array}{cc}\text { PG blastocysts, } & \text { PG ES lines derived, } \\ \boldsymbol{n} \text { (\% 2-cell embryos) } & \boldsymbol{n} \text { (\% blastocysts) } \\ 18(48.1) & 10(55.6) \\ 22(75.9), 25(75.8) & 8(36.4), 5(20)\end{array}$

AMultiple-result entries in the same column indicate results from independent derivation experiments.
PG ES lines heterozygous for $\boldsymbol{H} \boldsymbol{b} \boldsymbol{b}^{\text {th-4}}$ allele, $\boldsymbol{n}$ (\% lines) NA $10(76.9)$

PG ES lines without
$\boldsymbol{H b}^{\text {th-4 }}$ allele, $\boldsymbol{n}$ (\% lines)
NA
$3(23.1)$

$3(23.1)$ 

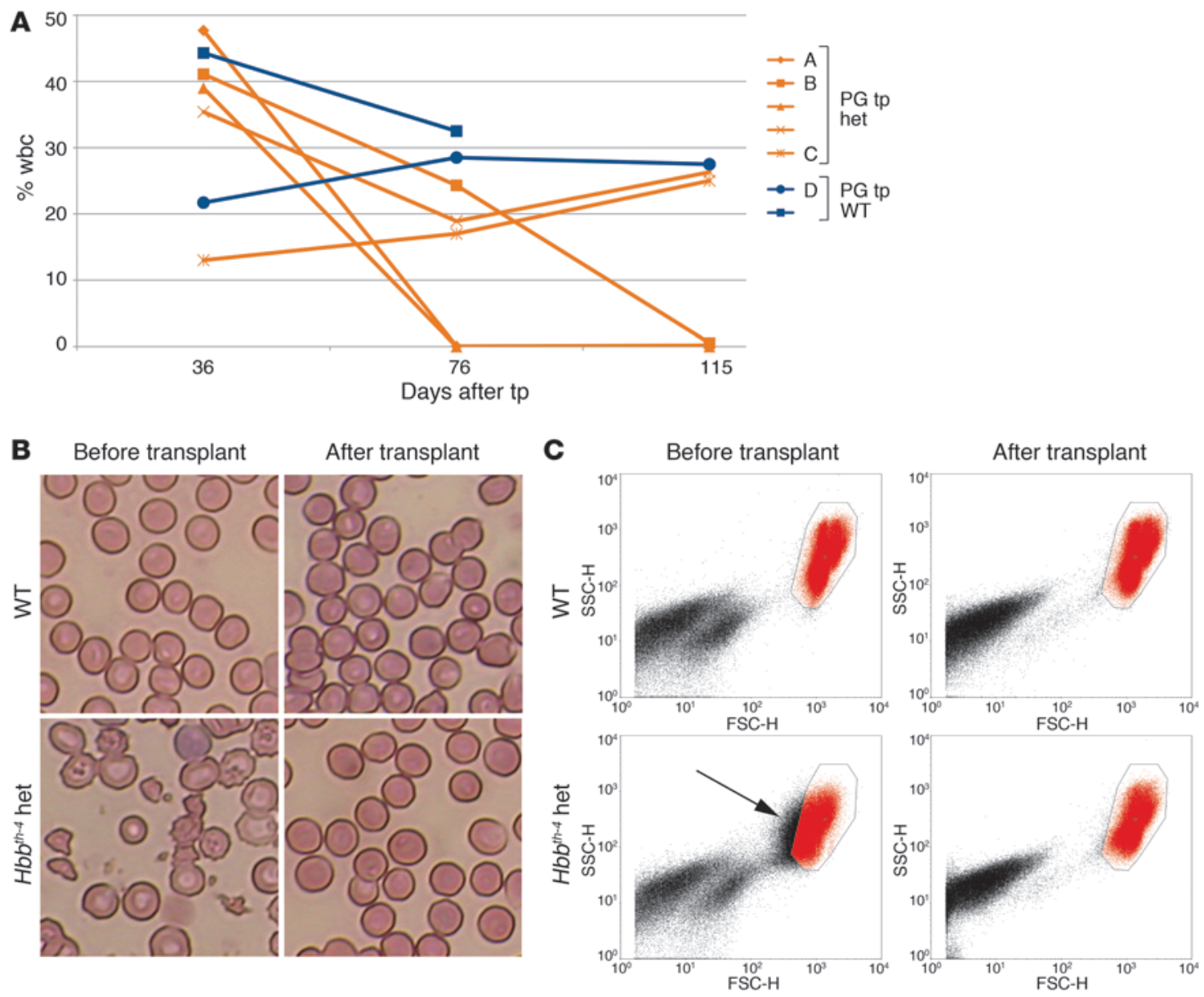

\section{Figure 2}

Engraftment of in vitro hematopoietic derivatives of autologous PG ES cells in $\mathrm{Hb} b^{t h-4} / \mathrm{Hbb}^{+}$mice corrects rbc phenotype. (A) Contribution of PG ES cell derivatives to peripheral wbc after transplantation (tp) into $H b b^{\text {th-4 }}$ heterozygous (het; yellow lines) and WT littermate (blue lines) recipients. Three recipients exhibited stable, long-term engraftment. (B) Blood smears of WT and heterozygous mice before and 4 weeks after transplantation of PG ES cell derivatives. Original magnification, $\times 40$. (C) Forward/side scatter plots of rbc (red) from WT and het mice before and 115 days after transplantation (arrow highlights cells outside a gate defined by properties of WT rbc).

to the parental strains of the hybrid oocyte donors (C57BL/6 and 129S1). Both lines were homozygous for $129 \mathrm{~S} 1$ markers at all loci tested centromeric to and including the MHC locus (10-37 million bp on chromosome 17, including 2 SNPs within the MHC [histocompatibility 2, blastocyst; and histocompatibility 2, $\mathrm{M}$ region locus 3]; Supplemental Figure 2C). Therefore, with the exception of minor histocompatibility antigens encoded elsewhere, both lines are thus MHC homozygous for 129S1-derived alleles.

Ectopic expression of the homeodomain protein HOXB4 in differentiating PG ES cell cultures was used to promote the formation of adult-engrafting hematopoietic progenitor/stem cells (25). After transplantation of ES cell-derived hematopoietic progenitor/stem cells from WT control (N) and $\mathrm{Hbb}^{\text {th-4 }}$ allele-free PG ES cells derived from heterozygous females into $(\mathrm{C} 57 \mathrm{BL} / 6 \times 129 \mathrm{SvEv}) \mathrm{F}_{1}$ (designated B6129SvEvF1) $\mathrm{Hbb}^{+} / \mathrm{Hbb}^{+}$(WT) and B6129SvEvF1 $\mathrm{Hbb}^{t h-4} / \mathrm{Hbb}^{+}$ (heterozygous) mice, recipients of both $\mathrm{N}$ and PG cell transplants exhibited engraftment of ES cell derivatives, with more than $40 \%$ and $20 \%$ contribution to peripheral wbc at 5 and 16 weeks after transplantation, respectively (Figure $2 \mathrm{~A}$ ). We did not observe rejection of PG ES cell lines homozygous for the 129S1 H-2 haplotype
$\left(\mathrm{H}-2^{\mathrm{bc}}\right)$ in hybrid recipients heterozygous for $129 \mathrm{SvEv}\left(\mathrm{H}-2^{\mathrm{bc}}\right)$ and $\mathrm{C} 57 \mathrm{BL} / 6$ haplotypes $\left(\mathrm{H}-2^{\mathrm{b}}\right)$. The $\mathrm{H}-2^{\mathrm{b}}$ and $\mathrm{H}-2^{\mathrm{bc}}$ haplotypes are considered identical in the classical region but differ in the $\mathrm{T}$ region, which could lead to graft-versus-host disease.

None of the recipients exhibited any pathology or tumor formation associated with the transplants. ES cell derivatives were present in the erythroid lineage (Ter119-positive, Supplemental Figure 3A), with minor contribution to lymphoid cells (data not shown), consistent with observations of pluripotent stem cell-derived hematopoietic transplants $(3,4,23,25)$. Analysis of mouse strain-specific variants of glucose phosphate isomerase 1 in total peripheral blood (99\% erythrocytes) revealed high levels of rbc replacement by ES cell derivatives. In several recipients with moderate contribution of ES cell derivatives to wbc, rbc were mostly ES cell-derived (Supplemental Figure 3B), suggesting selective donor-derived erythroid replacement. Transplants of in vitro hematopoietic ES cell derivatives in mouse models typically result in mixed chimerism $(3,23$, 25 ), as seen here; however, due to a putative selective advantage of the normal cells, this can be sufficient to reverse thalassemic phenotypes $(26,27) . H b b^{t h-4}$ heterozygotes with ES cell-derived engraft- 


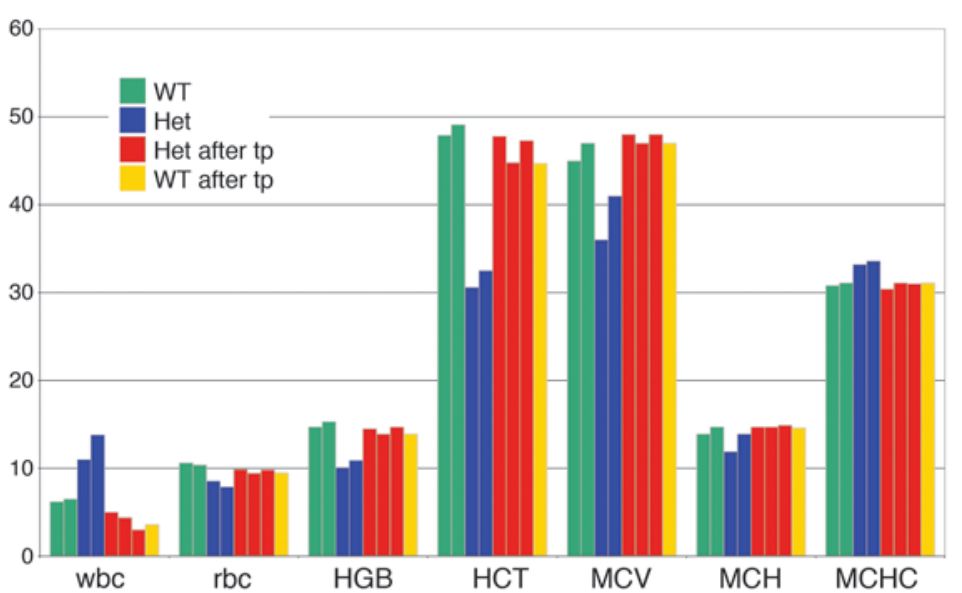

Figure 3

Improvement of hematologic values in transplanted thalassemic mice. Examples of values from nontransplanted WT and $\mathrm{Hbb}^{\text {th-4 }}$ heterozygous mice; and from WT and het recipients after transplantation of in vitro PG ES cell derivatives, with $30 \%-80 \%$ ES cell-derived contribution to wbc. wbc data are presented as $10^{3} / \mathrm{mm}^{3}$; rbc, as $10^{6} / \mathrm{mm}^{3}$; hemoglobin (HGB), g/dl; hematocrit (HCT), $\%$; mean corpuscular volume (MCV), fl; mean corpuscular hemoglobin ( $\mathrm{MCH}$ ), pg; mean corpuscular hemoglobin concentration (MCHC), g/dl.

ment exhibited normal rbc morphology (Figure 2B). Compared with the rbc population of normal mice with distinct forward and side scatter characteristics visualized by fluorescence-activated cell sorting (FACS), rbc from $\mathrm{Hb}^{\text {th-4 }}$ heterozygotes are characterized by a much broader distribution in size, apparent in the wider distribution in forward scatter (Figure 2C). Hbb $b^{\text {th-4 }}$ heterozygous recipients with engraftment of PG ES cell hematopoietic derivatives exhibited correction to an rbc size distribution similar to that in normal mice (Figure 2C). Evaluation of hematologic parameters revealed improvement of parameters that were abnormal in $\mathrm{Hbb}^{t h-4}$ heterozygotes, including low hematocrit, low rbc, and low mean corpuscular hemoglobin. These values were normal in heterozygous animals with engraftment of either autologous PG ES cell (Figure 3) or $\mathrm{N}$ ES cell-derived transplants (data not shown). Pathology indicated reduction of splenomegaly as well as absence of extramedullary hematopoiesis and iron deposits in spleen and kidney characteristic for $\mathrm{Hbb}^{t h-4}$ heterozygous animals (Supplemental Figure 3C, and data not shown). In summary, we observed long-term reversion of the thalassemic phenotype in $\mathrm{Hbb}^{\text {th-4 }}$ heterozygous recipient mice with engraftment of PG ES cell in in vitro hematopoietic derivatives. Consistent with previous observations, the absence of imprinting-associated phenotypes after transplantation of uniparental ES cell derivatives into the hematopoietic system may be due to inconsequential levels of expression of imprinted genes (23), but could also reflect selection of cells that are epigenetically more similar to normal cells (28).

The approach described here utilizes the incidence of homozygosity in PG ES cells in order to eliminate an unwanted allele present in the heterozygous gamete donor. We have previously demonstrated that at least for hematopoietic tissues, the epigenome of uniparental ES cell differentiated into blood does not limit the therapeutic outcome (23). The extent to which the increase in homozygosity may result in incidence of homozygosity at deleterious recessive alleles is unknown, but the estimate of lethal alleles in humans is surprisingly low (0.7 per gamete, ref. 29), based on consanguinity data; and the relevance of these recessive alleles would be predictably much lower in individual adult tissues.

Human pluripotent cells derived from embryos are potentially the optimal choice in terms of differentiation potential and safety, at least until the fidelity of reprogramming in iPS cells can be resolved (30). Gamete selection to generate zygotes and ES cells that are disease allele-free may be the only solution for large or complex deletions that are poorly defined or not amenable to current gene therapy approaches or in vitro gene modification. If they are MHC-homozygous, such PG ES cell lines could be immune compatible with the patient's offspring, and potentially with a wider proportion of the population (31). In the case of recessive diseases, MHC-homozygous, disease-free PG ES cells derived from donated oocytes from a heterozygous mother may also generate useful pluripotent cells for treatment in their affected offspring.

In summary, the derivation of uniparental ES cell lines can be utilized to obtain autologous stem cells devoid of undesirable alleles. An inverse approach, deriving ES cell lines with homozygosity of alleles heterozygous in the gamete donor, could also be employed to derive pluripotent stem cell lines homozygous for lethal alleles that can be used for in vitro assays and drug discovery.

\section{Methods}

$P G E S$ cell derivation and characterization. Animals were maintained and used for experimentation according to the guidelines of the Institutional Animal Care and Use Committee of the University of Pennsylvania. PG ES cell lines were derived from unfertilized MII oocytes from B6129S1F1 Hbb $b^{t h-4}$ heterozygous females as described previously (32) and characterized as described in Supplemental Methods.

Transplantation. ES cells were in vitro differentiated as described $(23,25)$, and $1 \times 10^{6}$ to $2 \times 10^{6}$ cells per recipient transplanted into lethally irradiated (9.5 Gy), NK-depleted (anti-asialo GM1 [Wako] i.p. injected 24 hours before transplantation) B6129SvEvF1 $\mathrm{Hbb}^{\text {th-4 }}$ heterozygous and WT mice.

Recipient analysis. ES cell contribution and blood lineage analyses were performed as described previously (23) and as described in Supplemental Methods. Forward and side scatter characteristics of rbc were determined by low-speed analysis (FACScan, BD) of peripheral blood diluted 1:50 into PBS. Peripheral blood hematology values were obtained using a SCIL analyzer.

\section{Acknowledgments}

We thank Terry Jordan for animal husbandry, Kimberly Jennings for assistance with genotyping, and Albrecht Müller for comments on the manuscript.

Received for publication October 8, 2010, and accepted in revised form November 17, 2010

Address correspondence to: K. John McLaughlin, Center for Molecular and Human Genetics, The Research Institute at Nationwide Children's Hospital, 700 Children's Drive, Columbus, Ohio 43205, USA. Phone: 614.355.3639; Fax: 614.722.2817; E-mail: john.mclaughlin@nationwidechildrens.org.

Seigo Hatada's present address is: Department of Surgery, CedarsSinai Medical Center, Los Angeles, California, USA. 
1. Fischer A, Cavazzana-Calvo M. Gene therapy of inherited diseases. Lancet. 2008;371(9629):2044-2047.

2. Kaiser J. Gene therapy. Beta-thalassemia treatment succeeds, with a caveat. Science. 2009; 326(5959):1468-1469.

3. Rideout WM, Hochedlinger K, Kyba M, Daley GQ, Jaenisch R. Correction of a genetic defect by nuclear transplantation and combined cell and gene therapy. Cell. 2002;109(1):17-27.

4. Hanna J, et al. Treatment of sickle cell anemia mouse model with iPS cells generated from autologous skin. Science. 2007;318(5858):1920-1923.

5. Giudice A, Trounson A. Genetic modification of human embryonic stem cells for derivation of target cells. Cell Stem Cell. 2008;2(5):422-433.

6. McGrath J, Solter D. Completion of mouse embryogenesis requires both the maternal and paternal genomes. Cell. 1984;37(1):179-183.

7. Barton SC, Surani MA, Norris ML. Role of paternal and maternal genomes in mouse development. Nature. 1984;311(5984):374-376.

8. Cattanach BM, Kirk M. Differential activity of maternally and paternally derived chromosome regions in mice. Nature. 1985;315(6019):496-498.

9. Robertson EJ, Kaufman MH, Bradley A, Evans MJ. Isolation, properties, and karyotype analysis of pluripotential (EK) cell lines from normal and parthenogenetic embryos. In: Silver LM, Martin GR, Strickland S, eds. Teratocarcinomal Stem Cells. Cold Spring Harbor Conferences on Cell Proliferation. New York, New York, USA: Cold Spring Harbor Laboratory; 1983:647-663.

10. Mann JR, Gadi I, Harbison ML, Abbondanzo SJ, Stewart CL. Androgenetic mouse embryonic stem cells are pluripotent and cause skeletal defects in chimeras: implications for genetic imprinting. Cell. 1990; 62(2):251-260

11. Song WJ, et al. Haploinsufficiency of CBFA2 causes familial thrombocytopenia with propensity to develop acute myelogenous leukaemia. Nat Genet. 1999;23(2):166-175.

12. Matheny CJ, et al. Disease mutations in RUNX1 and RUNX2 create nonfunctional, dominant-negative, or hypomorphic alleles. Embo J. 2007;26(4):1163-1175.

13. Montosi G, et al. Autosomal-dominant hemochromatosis is associated with a mutation in the ferroportin (SLC11A3) gene. J Clin Invest. 2001;108(4):619-623.

14. Germeshausen M, Skokowa J, Ballmaier M, Zeidler C, Welte K. G-CSF receptor mutations in patients with congenital neutropenia. Curr Opin Hematol. 2008; 15(4):332-337.

15. Hayward CP, et al. An autosomal dominant, qualitative platelet disorder associated with multimerin deficiency, abnormalities in platelet factor $\mathrm{V}$, thrombospondin, von Willebrand factor, and fibrinogen and an epinephrine aggregation defect. Blood. 1996;87(12):4967-4978.

16. Diamandis $M$, et al. Quebec platelet disorder is linked to the urokinase plasminogen activator gene (PLAU) and increases expression of the linked allele in megakaryocytes. Blood. 2009;113(7):1543-1546.

17. Rahman N, Scott RH. Cancer genes associated with phenotypes in monoallelic and biallelic mutation carriers: new lessons from old players. Hum Mol Genet. 2007;16 spec no 1:R60-R66.

18. Kim K, et al. Histocompatible embryonic stem cells by parthenogenesis. Science. 2007;315(5811):482-486.

19. Revazova ES, et al. Patient-specific stem cell lines derived from human parthenogenetic blastocysts. Cloning Stem Cells. 2007;9(3):432-449.

20. Mai Q, et al. Derivation of human embryonic stem cell lines from parthenogenetic blastocysts. Cell Res. 2007;17(12):1008-1019.

21. Revazova ES, et al. HLA homozygous stem cell lines derived from human parthenogenetic blastocysts. Cloning Stem Cells. 2008;10(1):11-24.

22. Lewis J, et al. A common human beta globin splicing mutation modeled in mice. Blood. 1998;
91(6):2152-2156.

23. Eckardt S, Leu NA, Bradley HL, Kato H, Bunting $\mathrm{KD}$, Mclaughlin KJ. Hematopoietic reconstitution with androgenetic and gynogenetic stem cells. Genes Dev. 2007;21(4):409-419.

24. Morgan TH. Random segregation versus coupling in mendelian inheritance. Science. 1911;34(873):384.

25. Kyba M, Perlingeiro RC, Daley GQ. HoxB4 confers definitive lymphoid-myeloid engraftment potential on embryonic stem cell and yolk sac hematopoietic progenitors. Cell. 2002;109(1):29-37.

26. Roberts C, Kean L, Archer D, Balkan C, Hsu LL. Murine and math models for the level of stable mixed chimerism to cure beta-thalassemia by nonmyeloablative bone marrow transplantation. Ann NY Acad Sci. 2005;1054:423-428.

27. Bethel CA, Murugesh D, Harrison MR, Mohandas N, Rubin EM. Selective erythroid replacement in murine beta-thalassemia using fetal hematopoietic stem cells. Proc Natl Acad Sci U S A. 1993; 90(21):10120-10124.

28. Li C, et al. Correlation of expression and methylation of imprinted genes with pluripotency of parthenogenetic embryonic stem cells. Hum Mol Genet. 2009;18(12):2177-2187.

29. Bittles AH, Neel JV. The costs of human inbreeding and their implications for variations at the DNA level. Nat Genet. 1994;8(2):117-121.

30. Kim K, et al. Epigenetic memory in induced pluripotent stem cells. Nature. 2010;467(7313):285-290.

31. Nakajima F, Tokunaga K, Nakatsuji N. Human leukocyte antigen matching estimations in a hypothetical bank of human embryonic stem cell lines in the Japanese population for use in cell transplantation therapy. Stem Cells. 2007;25(4):983-985.

32. Eckardt S, McLaughlin KJ. Production of uniparental embryonic stem cell lines. In: Baharvand $\mathrm{H}$, ed. Trends in Stem Cell Biology and Technology. New York, New York, USA: Humana Press; 2009:19-38. 